The published version of this journal can be found at: https://doi.org/10.1038/s41584018-0076-7 : https://rdcu.be/6UJC

\title{
A Notch into the joint that exacerbates osteoarthritis
}

\section{News and Views article}

Silvia Monteagudo ${ }^{1}$ and Rik J. Lories ${ }^{1,2}$

${ }^{1} \mathrm{KU}$ Leuven, Department of Development and Regeneration, Skeletal Biology and Engineering Research Center, Laboratory of Tissue Homeostasis and Disease

${ }^{2}$ UZ Leuven, Division of Rheumatology

Correspondence: Rik.Lories@kuleuven.be

Increasing evidence supports the view that postnatal joint homeostasis and prevention of osteoarthritis, the most common type of chronic joint disease, is dependent on tight regulation of signaling cascades with essential roles in joint and bone development. The importance of the transforming growth factor (TGF) superfamily, including bone morphogenetic proteins (BMPs) and the Wnt signaling cascades, have been demonstrated in multiple in vitro and in vivo models, and agonists or antagonists of these cascades are evaluated in early phase clinical trials ${ }^{1,2}$. Notch signaling is another key signaling mechanism that controls cell fate, differentiation and function in a wide range of tissues from development to disease ${ }^{3}$.

Mutations in the Notch signaling pathway can lead to a wide variety of clinical syndromes. Among these, the Hadju Cheney Syndrome (HCS) is a rare disorder in which the skeletal abnormalities are most striking ${ }^{4}$. The patients demonstrate extensive bone loss, systemically leading to osteoporotic fractures and locally to acro-osteolysis with resorption of the distal phalanges of hands and feet. HCS is caused by gain-of-function mutations in the NOTCH2 gene. The mutation introduces a stop codon that results in the deletion of the terminal PEST domain that targets the molecule's degradation in the proteasome. Mutations resulting in syndromes often provide useful information about a gene's function. The team of E. Canalis has developed a mouse strain carrying this HCS-type gain-of-function mutation and studied its effect on osteoarthritis triggered by joint injury ${ }^{5}$.

The experiments demonstrated that Notch 2 mutant mice are effectively characterized by increased NOTCH2 signaling as studied by target gene expression analysis of femoral heads ${ }^{5}$. The knee joints of these mice were further evaluated in the destabilization of the medial meniscus (DMM) model of osteoarthritis, in which transection of the medial meniscotibial ligament results in mild instability and mild to moderate osteoarthritis over a course of 2 to 3 months. Whereas no differences in sham-operated joints were found between the male mutant mice and their wild-type littermates, DMM surgery did result in increased severity of disease in the Notch 2 gain-of-function animals, that was characterized by increased cartilage damage, thicker subchondral bone and larger osteophytes ${ }^{5}$. These data suggest that 
increased levels or activity of NOTCH2 may contribute to the severity of osteoarthritis upon joint trauma.

The Notch signaling cascade appears to be relatively simple but remarkable. In contrast to most signaling cascades, Notch signaling is largely dependent on cell-cell communication as ligands (the Jagged and Delta-like family in mammals) as well as receptors (Notch 1-4 in mammals) are transmembrane proteins ${ }^{3}$. Upon ligand-receptor interaction, the Notch receptor is cleaved by two enzymes (a metalloproteinase and a gamma-secretase complex), resulting in the cleavage of the Notch Intracellular Domain (NICD) that translocates into the nucleus and forms a complex at the chromatin level with recombination signal binding protein for immunoglobulin kappa J region (RBPJ) and mastermind-like protein (MAML), thereby triggering the activation of specific transcriptional responses ${ }^{3}$. The different Notch receptors and ligand-interactions, distinct repertoires of modifier molecules at all levels of the cascade including the transcriptional complexes, the epigenetic status of the target genes, and the cross-talk with other signaling pathways, are all considered factors that contribute to the diversity in signaling outcome, and are an area for extensive further research ${ }^{3}$.

The focus on Notch2 in the recent paper ${ }^{5}$ is of particular interest as data with regards to the Notch signaling cascade in joint biology have been confusing and may seem to be contradictory. In development, mouse genetic approaches targeting either the Notch1 receptor or the nuclear co-factor RBPJ show that changing the balance of Notch signaling affects the normal differentiation process of the developmental and growth plate chondrocytes from proliferation to hypertrophy 6,7 . The observed abnormalities are dependent on the stage and target of the genetic recombination during the endochondral bone formation process. In the context of osteoarthritis, postnatally induced cartilage-specific deletion of RBPJ using a type II collagen driven tamoxifen-dependent Cre-mouse protected the joint against surgically induced osteoarthritis in one set of experiments ${ }^{7}$, whereas a similar setup using either a type II collagen driven or an aggrecan promotor driven tamoxifendependent Cre-mouse led to the spontaneous development of osteoarthritis, a striking difference that remains unexplained ${ }^{8-10}$. By targeting Notch2 specifically, the current report confers a level of specificity that is absent in the RBPJ knockout animals ${ }^{5}$. Effectively, loss of $R B P J$ will block downstream signals of all Notch receptors and could also have additional effects on transcription. In the absence of the NICD-MAML-RBPJ interaction, RBPJ likely acts as a transcriptional suppressor and its deletion may therefore have effects that are not directly related to the activation of Notch signaling ${ }^{3}$.

Gain-of-function approaches towards the other Notch receptors may provide further insights into the role of this cascade in osteoarthritis. In addition, several other questions remain to be addressed. The specific interaction of ligands and receptors in Notch signaling is considered to be dependent on cell-cell contact, a feature that is not obvious in the context of the articular cartilage where the cells are dispersed in their self-produced pericellular and intercellular matrix. Alternative modes of action could be the presence of cell extensions or filopodia that are responsible for the cell-cell contact, or the use of exosomes within the cartilage. In addition, there is some evidence that the ligand-receptor interaction of the Notch cascade is not limited to cell-cell interaction but can also take place within the cell ${ }^{3}$. The latter phenomenon would be of specific interest in the articular chondrocytes. Finally, the upregulation of interleukin-6, proposed by Zanotti et al. as mechanism contributing to 
osteoarthritis in the mutant mice, could result from an interaction of the Notch signaling with other cascades such as the NFkB signaling pathway, and may not be dependent on the NICDMAML-RBPJ interaction in the commonly known pathway ${ }^{3}$.

In conclusion, the observation that a gain-of-function mutation in the Notch2 gene shifts the balance of this signaling pathway towards hyper-activation and results in more severe osteoarthritis in surgically challenged mice, supports the view that tight regulation of cascades associated with skeletal development is essential to maintain joint homeostasis in adult life. This way, Notch signaling appears to follow the path of Wnt and transforming growth factor beta signaling ${ }^{1,2}$.

\section{Acknowledgements and conflict of interests}

Original research on osteoarthritis in the Laboratory of Tissue Homeostasis and Disease is supported by Excellence of Science Grant "Join-t-against-OA", grants from the Flanders Research Foundation (FWO Vlaanderen), a C1 grant from KU Leuven and an unrestricted grant from Celgene to Dr. Monteagudo. Dr. Monteagudo was the recipient of a Marie-Curie fellowship named "DOTOS". Leuven Research and Development, the technology transfer office of KU Leuven has received consultancy and speakers fees on behalf of R.J.L from Galapagos and Samumed, active in the field of osteoarthritis.

\section{Box 1: rising questions with regards to the role of Notch signaling in osteoarthritis}

- Specific roles of the different Notch receptors and their intracellular signaling domain in the different tissues of the joint (cartilage, bone and synovium).

- Individual ligand - receptor interactions, their effects on the amplitude and the specificity of the cell's molecular response.

- Interaction of Notch signaling in the joint tissues with other key signaling cascades such as TGF/BMP and Wnt signaling, and the relationship between Notch and hypoxia factors.

- The roles of intercellular (trans) and intracellular ligand-receptor (cis) interactions in particular in extra-cellular matrix rich regions such as cartilage and bone

- Importance of the canonical RBPJ dependent pathway versus activation of alternative cascades.

\section{References}

1 Monteagudo, S. \& Lories, R. J. Cushioning the cartilage: a canonical Wnt restricting matter. Nat Rev Rheumatol 13, 670-681, doi:10.1038/nrrheum.2017.171 (2017).

2 van der Kraan, P. M. The changing role of TGFbeta in healthy, ageing and osteoarthritic joints. Nat Rev Rheumatol 13, 155-163, doi:10.1038/nrrheum.2016.219 (2017).

3 Siebel, C. \& Lendahl, U. Notch Signaling in Development, Tissue Homeostasis, and Disease. Physiol Rev 97, 1235-1294, doi:10.1152/physrev.00005.2017 (2017).

4 Canalis, E. Clinical and experimental aspects of notch receptor signaling: HajduCheney syndrome and related disorders. Metabolism 80, 48-56, doi:10.1016/j.metabol.2017.08.002 (2018). 
5 Zanotti, S., Yu, J., Bridgewater, D., Wolf, J. M. \& Canalis, E. Mice harboring a Hajdu Cheney Syndrome mutation are sensitized to osteoarthritis. Bone 114, 198-205, doi:10.1016/j.bone.2018.06.020 (2018).

6 Kohn, A. et al. Cartilage-specific RBPjkappa-dependent and -independent Notch signals regulate cartilage and bone development. Development 139, 1198-1212, doi:10.1242/dev.070649 (2012).

7 Hosaka, Y. et al. Notch signaling in chondrocytes modulates endochondral ossification and osteoarthritis development. Proc Natl Acad Sci U S A 110, 1875-1880, doi:10.1073/pnas.1207458110 (2013).

8 Liu, Z. et al. Notch signaling in postnatal joint chondrocytes, but not subchondral osteoblasts, is required for articular cartilage and joint maintenance. Osteoarthritis Cartilage 24, 740-751, doi:10.1016/j.joca.2015.10.015 (2016).

9 Liu, Z. et al. A dual role for NOTCH signaling in joint cartilage maintenance and osteoarthritis. Sci Signal 8, ra71, doi:10.1126/scisignal.aaa3792 (2015).

10 Mirando, A. J. et al. RBP-Jkappa-dependent Notch signaling is required for murine articular cartilage and joint maintenance. Arthritis Rheum 65, 2623-2633, doi:10.1002/art.38076 (2013). 